\title{
Arrestin Translocation Is Induced at a Critical Threshold of Visual Signaling and Is Superstoichiometric to Bleached Rhodopsin
}

\author{
Katherine J. Strissel, ${ }^{1}$ Maxim Sokolov, ${ }^{1,2}$ Lynn H. Trieu, ${ }^{1}$ and Vadim Y. Arshavsky ${ }^{1,3}$ \\ ${ }^{1}$ Department of Ophthalmology, Harvard Medical School and the Massachusetts Eye and Ear Infirmary, Boston, Massachusetts 02114, ${ }^{2}$ Department of \\ Ophthalmology, West Virginia University and the West Virginia University Eye Institute, Morgantown, West Virginia 26506, and ${ }^{3}$ Departments of \\ Ophthalmology and Neurobiology, Duke University, Durham, North Carolina 27710
}

\begin{abstract}
Light induces massive translocation of major signaling proteins between the subcellular compartments of photoreceptors. Among them is visual arrestin responsible for quenching photoactivated rhodopsin, which moves into photoreceptor outer segments during illumination. Here, for the first time, we determined the light dependency of arrestin translocation, which revealed two key features of this phenomenon. First, arrestin translocation is triggered when the light intensity approaches a critical threshold corresponding to the upper limits of the normal range of rod responsiveness. Second, the amount of arrestin entering rod outer segments under these conditions is superstoichiometric to the amount of photoactivated rhodopsin, exceeding it by at least 30-fold. We further showed that it is not the absolute amount of excited rhodopsin but rather the extent of downstream cascade activity that triggers translocation. Finally, we demonstrated that the total amount of arrestin in the rod cell is nearly 10 -fold higher than previously thought and therefore sufficient to inactivate the entire pool of rhodopsin at any level of illumination. Thus, arrestin movement to the outer segment leads to an increase in the free arrestin concentration and thereby may serve as a powerful mechanism of light adaptation.
\end{abstract}

Key words: arrestin; photoreceptor; protein translocation; rhodopsin; vision; retina

\section{Introduction}

Vertebrate rods and cones have a remarkable ability to adapt their physiological responses to ever-changing conditions of ambient illumination. Multiple molecular and cellular mechanisms underlie light adaptation, which serves to optimize the response speed and sensitivity of the cell to operate at various light intensities (for review, see Burns and Baylor, 2001; Fain et al., 2001; Arshavsky et al., 2002). One adaptation mechanism consists of massive light-dependent translocation of signaling proteins into and out of the photoreceptor outer segment in which phototransduction takes place (for review, see Arshavsky, 2003; Hardie, 2003; Strissel et al., 2004). Three translocating proteins have been identified so far: arrestin, responsible for quenching photoactivated rhodopsin $\left(\mathrm{R}^{*}\right)$ (Broekhuyse et al., 1985; Philp et al., 1987; Mangini and Pepperberg, 1988; Whelan and McGinnis, 1988; Zhu et al., 2002; Mendez et al., 2003; Peterson et al., 2003; Zhang et al., 2003a,b; Peet et al., 2004; Coleman and Semple-Rowland, 2005; Nair et al., 2005); recoverin, a $\mathrm{Ca}^{2+}$-binding protein also regulating the lifetime of activated rhodopsin (Strissel et al.,

\footnotetext{
Received 0ct. 7, 2005; revised Dec. 1, 2005; accepted Dec. 12, 2005.

This work was supported by the National Institutes of Health Grant R01 EY10336, National Institutes of Health Core Grant for Vision Research EY14104, and a Massachusetts Lions Eye Research Fund grant (V.Y.A.).

Correspondence should be addressed to Vadim Arshavsky, Department of Ophthalmology, Duke University Medical Center, 5008 Albert Eye Research Institute, 2351 Erwin Road, Durham, NC 27710. E-mail: vadim.arshavsky@duke.edu.

DOI:10.1523/JNEUROSCI.4289-05.2006

Copyright $\odot 2006$ Society for Neuroscience $\quad 0270-6474 / 06 / 261146-08 \$ 15.00 / 0$
}

2005); and transducin, the G-protein mediating phototransduction (Brann and Cohen, 1987; Philp et al., 1987; Whelan and McGinnis, 1988; Pulvermüller et al., 2002; Sokolov et al., 2002; Mendez et al., 2003; Zhang et al., 2003b; Elias et al., 2004; Kassai et al., 2005; Nair et al., 2005). In the light, arrestin moves into the outer segments, whereas transducin and recoverin move in the opposite direction. Overall, these protein rearrangements are thought to lead to a reduction in photoreceptor sensitivity and to the shortening of response duration, hallmarks of light adaptation.

The phenomenon of protein translocation has drawn overwhelming interest in recent years; however, the underlying molecular and cellular mechanisms remain far from being understood. Most mechanistic studies have been devoted to determining whether translocation is driven by molecular motors or diffusion (compare McGinnis et al., 2002 with Nair et al., 2005) (for review, see Strissel et al., 2004). In the case of arrestin, one straightforward hypothesis is that its redistribution in light arises passively by the binding by arrestin to photoactivated rhodopsin in the outer segment (Mangini et al., 1994; Nair et al., 2005). A key prediction of this hypothesis is that the amount of the translocated arrestin is equal to the amount of activated rhodopsin, but such quantitation has never before been accomplished. Here we report a comprehensive quantitative analysis of arrestin translocation in mouse rods, which revealed that the amount of translocating arrestin significantly exceeds the amount of photoactivated rhodopsin. Furthermore, arrestin 
translocation is triggered only when the illumination exceeds a threshold intensity. Finally, using transgenic mice in which phototransduction is either enhanced [RGS9 anchor protein (R9AP) knock-out] or abolished (transducin $\alpha$ subunit knock-out), we find that triggering translocation requires a critical level of phototransduction signaling downstream from rhodopsin and is not dependent on the absolute amount of rhodopsin excited by light.

\section{Materials and Methods}

Animals. C57BL/6 wild-type mice were purchased from Charles River Laboratories (Wilmington, MA). R9AP knock-out mice were described previously by Keresztes et al. (2004). Transducin $\alpha$ subunit knock-out mice described by Calvert et al. (2000) were a gift from J. Lem (Tufts University, Boston, MA). Arrestin knock-out mice described by Xu et al. (1997) were a gift from J. Chen (University of Southern California, Los Angeles, CA). All animals were housed in a $12 \mathrm{~h}$ light/dark cycles and used between the ages of 2 and 3 months.

Serial sectioning with Western blotting. The method was originally described by Sokolov et al. (2002) and optimized for mice by Sokolov et al. (2004). Briefly, eyes were removed, the anterior portion was cut away, and the posterior eyecup was placed immediately into ice-chilled Ringer's solution. A $2 \mathrm{~mm}$ disc of central retina tissue was cut from the center of the eyecup with a surgical trephine and placed on a polyvinylidene difluoride membrane with the photoreceptors facing up. The sample was then flattened and frozen between two glass slides separated by $0.3 \mathrm{~mm}$ spacers. The base slide to which the retina was attached was then aligned for tangential sectioning at the microtome specimen holder. Serial sections, $5 \mu \mathrm{m}$ thick, were collected in a precooled $0.5 \mathrm{ml}$ Eppendorf (Westbury, NY) tube and stored at $-80^{\circ} \mathrm{C}$ until used. Western blotting was performed also as by Sokolov et al. (2004). Blots were probed with specific antibodies against arrestin (FC41; provided by L. Dinoso, Wills Eye Hospital, Philadelphia, PA) and the intracellular markers rhodopsin (4D2 provided by R. S. Molday, University of British Columbia, Vancouver, British Columbia, Canada) and cytochrome $c$ (H-104; Santa Cruz Biotechnology) and visualized using the Enhanced chemifluorescence (ECF) Detection System (Amersham Biosciences, Arlington Heights, IL). Note that the ECF detection system typically provides a better signal linearity than a more commonly used ECL system. The fluorescence of the bands was quantified using the Storm $860 \mathrm{Gel}$ and Blot Imaging System (Molecular Dynamics, Sunnyvale, CA).

To calculate the fraction of arrestin present in rod outer segments, we used the approach originally developed for calculating the outer segment fraction of transducin (Sokolov et al., 2002). The density of the immunostained bands in each section was plotted as a percentage of the total density of all bands. Those sections that contained rhodopsin but not cytochrome $c$ were considered as sections containing noncontaminated rod outer segment material. The fractions of arrestin and rhodopsin present in these sections were calculated, and the amount of arrestin in the entire outer segment was derived by taking their ratio.

Because the amount of arrestin in the noncontaminated rod outer segment sections obtained from the dark-adapted animals lies below the detectability limit of the ECF detection system, it is important to calculate the upper estimate of the arrestin content in those sections. Dark profiles (see Fig. 1) yield between 11 and 15 sections containing measurable amounts of arrestin. Although the amount of arrestin in individual sections varies, the experimental conditions are chosen in such a way that the density averages around the middle of the linear range of the ECF method (see Fig. 2), which depicts at least fourfold larger amount of arrestin than that falling below the detectability limit. A typical dark profile also yields at least one section containing not less than onequarter of total rhodopsin (and therefore rod outer segment material) and no detectible arrestin. Based on the above estimate of the detectability limit, the amount of arrestin in this section is $<25 \%$ of the average amount in the inner segment, and therefore the amount of arrestin in the entire outer segment is not more than the average value in one inner segment section. Thus, the most conservative estimate of the arrestin content in the dark-adapted rod outer segments is just under $7 \%$ of its total cellular content.
Light adaptation of animals and determination of rhodopsin bleaching levels. Mice were dark adapted for at least $12 \mathrm{~h}$ and anesthetized with an intraperitoneal injection of a ketamine/xylazine mixture (75 and $10 \mathrm{mg} /$ $\mathrm{kg}$, respectively), and their pupils were dilated with a mixture of $1 \%$ cyclopentolate- $\mathrm{HCl}, 2.5 \%$ phenylephrine, and $0.25 \%$ tropicamide. Light was delivered to the eyes by fiber-optic guides from an adjustable light source equipped with a $100 \mathrm{~W}$ halogen bulb. Even illumination throughout the entire retina was achieved by positioning a small white dome between the light guide and each of the eyes, just above the cornea surface, with drops of Gonak (Akorn, Buffalo Grove, IL) sealing the eye surface from exposure to air. The light intensity at the position corresponding to the eye surface was measured by a calibrated photodiode with a spectral sensitivity closely matched to that of rhodopsin. The photodiode was attached to a PDA-700 amplifier (TTI, Oriskany, NY). Mice were subjected to light exposure of a desired duration and light intensity.

The rate of rhodopsin photoactivation in rods was measured by determining the amount of bleached rhodopsin after illumination by light of known intensity. This was achieved by measuring rhodopsin concentration in the retina homogenate by difference spectroscopy before and after rhodopsin regeneration with 11-cis-retinal. A protocol modified from Sokolov et al. (2002) was used as follows. The retina was removed from the eye under dim red illumination and sonicated in $250 \mu$ of water. A $100 \mu \mathrm{l}$ aliquot was mixed with $20 \mu \mathrm{l}$ of $200 \mathrm{~mm}$ hydroxylamine (titrated to $\mathrm{pH} 7.5$ by $\mathrm{NaOH}$ ) containing $10 \% n$-octyl- $\beta$-D-glucopyranoside. The sample was centrifuged in a tabletop microcentrifuge, and rhodopsin concentration in the supernatant was measured by difference spectrometry using the molar extinction coefficient of 40,500 (Bownds et al., 1971). The rest of the sonicated sample was mixed with $0.5 \mu \mathrm{l}$ of $4 \mathrm{~mm}$ 11-cis-retinal solubilized in ethanol, sonicated again, and incubated at $37^{\circ} \mathrm{C}$ for $40 \mathrm{~min}$ in complete darkness. After the completion of regeneration, rhodopsin concentration was determined in a $100 \mu \mathrm{l}$ aliquot, as in the nonregenerated sample. The extent of rhodopsin bleaching in the retina was calculated as the difference between the measured rhodopsin concentration in regenerated and nonregenerated samples. For bleaching $<10 \%$ rhodopsin, rhodopsin bleaching to the desired level was achieved by reducing the intensity of calibrated light by neutral density filters. The conversion between the fraction of bleached rhodopsin and the rate of rhodopsin isomerization was performed assuming that the total amount of rhodopsin molecules in a mouse rod is $7 \times 10^{7}$ (Lyubarsky et al., 2004).

Expression and purification of recombinant mouse arrestin. A pProEX HTa plasmid (Invitrogen, Carlsbad, CA) containing the coding sequence of mouse visual arrestin was a gift from V. V. Gurevich and S. M. Hanson (Vanderbilt University, Nashville, TN). It was expressed in Escherichia coli according to standard procedures and purified from the inclusion bodies after a $2 \mathrm{~h}$ isopropyl- $\beta$-D-thiogalactopyranoside induction. Inclusion bodies were isolated by centrifugation $(30,000 \times \mathrm{g}$, for $10 \mathrm{~min})$ from cells disrupted by sonication in the presence of a protease inhibitor cocktail (Sigma, St. Louis, MO) and $10 \mathrm{~mm}$ DTT. Inclusion bodies were dissolved in $100 \mathrm{~mm} \mathrm{NaH}_{2} \mathrm{PO}_{4}, 10 \mathrm{~mm}$ Tris, and $6 \mathrm{~m}$ urea, $\mathrm{pH}$ 8.0, and incubated with Ni-NTA agarose beads (Qiagen, Hilden, Germany) for $1 \mathrm{~h}$ at $4^{\circ} \mathrm{C}$. The beads were washed three times with an excess of $100 \mathrm{~mm}$ $\mathrm{NaH}_{2} \mathrm{PO}_{4}, 10 \mathrm{~mm}$ Tris, and $6 \mathrm{M}$ urea, $\mathrm{pH} 6.3$, and arrestin was eluted with $100 \mathrm{~mm} \mathrm{NaH}_{2} \mathrm{PO}_{4}, 10 \mathrm{~mm}$ Tris, and $6 \mathrm{~m}$ urea, $\mathrm{pH}$ 4.5. Additional arrestin purification was performed on a Mono Q HR 16/10 column (Amersham Biosciences, Piscataway, NJ) in $20 \mathrm{~mm}$ Tris $\mathrm{HCl}, \mathrm{pH} 7.8$, and $6 \mathrm{~m}$ urea using a linear $0-1.0 \mathrm{M} \mathrm{NaCl}$ gradient. At least $95 \%$ pure arrestin was eluted in a single peak at $\sim 0.5 \mathrm{M} \mathrm{NaCl}$. Arrestin concentration was determined spectrophotometrically using its calculated molar extinction coefficient of $\epsilon_{280}=22,810$ or by the Bradford assay (Bradford, 1976) using bovine serum albumin as the standard, with both methods yielding essentially identical results.

\section{Results}

\section{Arrestin translocation is triggered as light intensity reaches a critical threshold}

To quantify the subcellular distribution of arrestin in the dark and the light dependence of its translocation, we used an ap- 


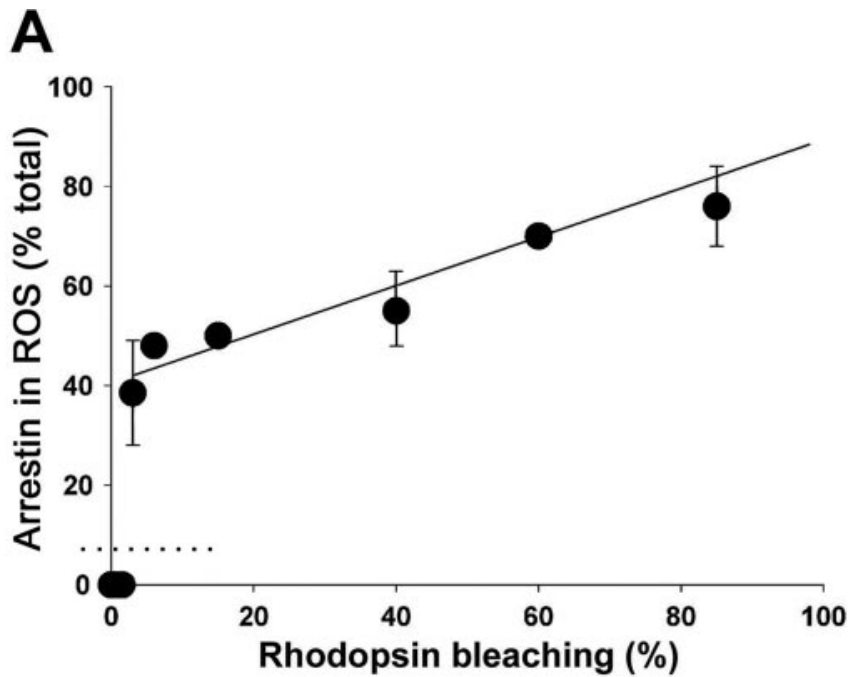

B

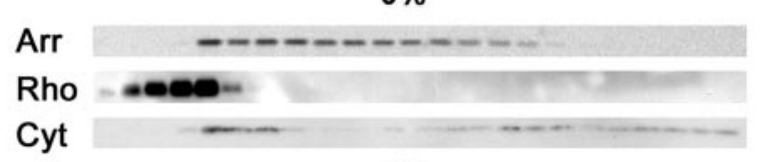

$3 \%$

Arr - - - - - - - - -

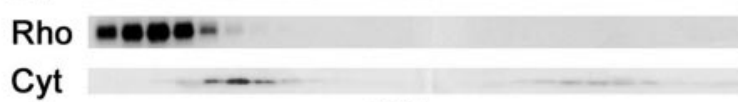

Cyt $80 \%$

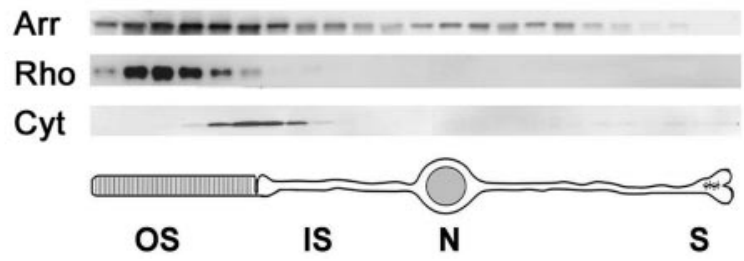

Figure 1. Light dependency of arrestin translocation. $\boldsymbol{A}$, Arrestin distribution in the rods of anesthetized mice kept in the dark or after 30 min of steady illumination at various intensities was analyzed by serial sectioning/Western blotting. The amount of arrestin in rod outer segments (ROS) was calculated as described in Materials and Methods. The amount of bleached rhodopsin at the end of the illumination period was measured by difference spectroscopy (see Materials and Methods). The most crucial measurements at $0,1.5$, and $3 \%$ rhodopsin bleaching levels were repeated five times, and other measurements were conducted between one and three times. The error bars represent SEM. The data of measurements at higher than $3 \%$ rhodopsin bleach levels are fitted by a straight line. The data at $0,0.5$, and $1.5 \%$ rhodopsin bleaching yielded an arrestin content in the outer segments below the most conservative estimate of the reliable detectability limit of the serial sectioning/Western blotting technique (dotted line at $7 \%$ arrestin; see Materials and Methods). B, Examples of the individual Western blot profiles of arrestin distribution throughout the serial sections in the dark (0\%) and at light intensities bleaching 3 and $80 \%$ rhodopsin. The arrestin distribution in each case is compared with those of two intracellular markers, rhodopsin (a marker for outer segments) and cytochrome c (a mitochondrial marker for inner segments). Below is shown a schematic drawing of a rod cell. Arr, Arrestin; Rho, rhodopsin; Cyt, cytochrome c; OS, outer segment; IS, inner segment; $N$, nucleus; $S$, synaptic terminal.

proach combining serial tangential sectioning of the retina with protein detection in each section by Western blotting. This method was originally developed for quantitative studies of transducin translocation (Sokolov et al., 2002). The dependency of arrestin translocation on the intensity of steady illumination (white light, $30 \mathrm{~min}$ ) is shown in Figure 1. In the dark, the rod outer segment content of arrestin is very low and below the detection limit of the serial sectioning/Western blotting technique, which we estimate conservatively not to exceed $7 \%$ of the total arrestin amount in the cell. Continuous light producing up to $\sim 580 \mathrm{R} * / \mathrm{rod} / \mathrm{s}$ (bleaching up to $1.5 \%$ rhodopsin during the entire illumination period) did not cause any detectable arrestin translocation. However, just doubling the light intensity to produce $\sim 1160 \mathrm{R}^{*} / \mathrm{rod} / \mathrm{s}$ (bleaching $3 \%$ rhodopsin during the experiment) triggered the translocation of $\sim 36 \%$ of the total arrestin pool. Light of higher intensities caused additional arrestin translocation, with the amount of moving arrestin approximately proportional to the amount of $\mathrm{R}^{\star}$ produced.

The light intensity threshold revealed in this experiment is striking. Potentially, it could be explained by either the accumulation of a critical amount of $\mathrm{R}^{\star}$ for arrestin binding or triggering a light-dependent signaling mechanism downstream from $R^{\star}$. A distinction between these possibilities requires the determination of the ratio between the amounts of photoexcited rhodopsin and translocated arrestin at the threshold intensity. Although rhodopsin bleaching can be measured with high precision by difference spectroscopy (see Materials and Methods), calculation of the number of moving arrestin molecules requires knowledge of its total molar content in the rod cell. This content was determined in the next set of experiments.

\section{The amount of arrestin in the entire rod cell is comparable with rhodopsin}

Most published arrestin measurements were conducted with preparations of isolated rod outer segments, in which arrestin content is typically determined to be $\sim 10 \%$ of rhodopsin (for a review on rod outer segment protein content, see Pugh and Lamb, 2000). However, because it is now generally accepted that most of the arrestin is located in the inner segments of darkadapted rods, the total arrestin content in the rod is expected to be larger. Indeed, an early report of the arrestin content in the whole retina suggested that the molar ratio between arrestin and rhodopsin was considerably higher (Broekhuyse et al., 1985), but this value was not generally accepted primarily because the electroimmunoassay technique used in that study could provide only rough estimates.

We determined the total content of arrestin in rods by quantitative Western blotting (Fig. 2). Because the rod-specific isoform of visual arrestin is absent in all other cell types of the retina, whole mouse retinas were used for this measurement. The amount of arrestin was calculated as a molar fraction of rhodopsin, which is a conventional approach for quantification of rodspecific proteins. Retinas obtained from dark-adapted mice were disrupted by sonication, and their rhodopsin content was determined by difference spectroscopy. The arrestin/rhodopsin molar ratio in these samples was then analyzed by Western blotting using purified recombinant mouse arrestin as the standard. To control for any potential artifacts related to quantitative immunoblotting in the presence of crude cellular homogenates, arrestin standards were combined with retina homogenates obtained from arrestin knock-out mice containing the same amount of rhodopsin as the wild-type retina samples. We found that the total amount of arrestin in rods is $78 \pm 1 \%$ (SEM; $n=6$ ) of rhodopsin (equivalent to $\sim 5.5 \times 10^{7}$ arrestin molecules, given $\sim 7 \times 10^{7}$ rhodopsin molecules in a mouse rod from Lyubarsky et al., 2004), which is much higher than the commonly assumed $10 \%$ of rhodopsin value and enough to quench all $\mathrm{R}^{\star}$ produced at nearly all light intensities (although our data do not directly imply that all of the rhodopsin molecules in a rod could in fact be phosphorylated and arrestin bound). 
A

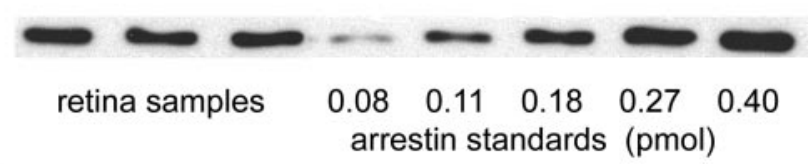

B

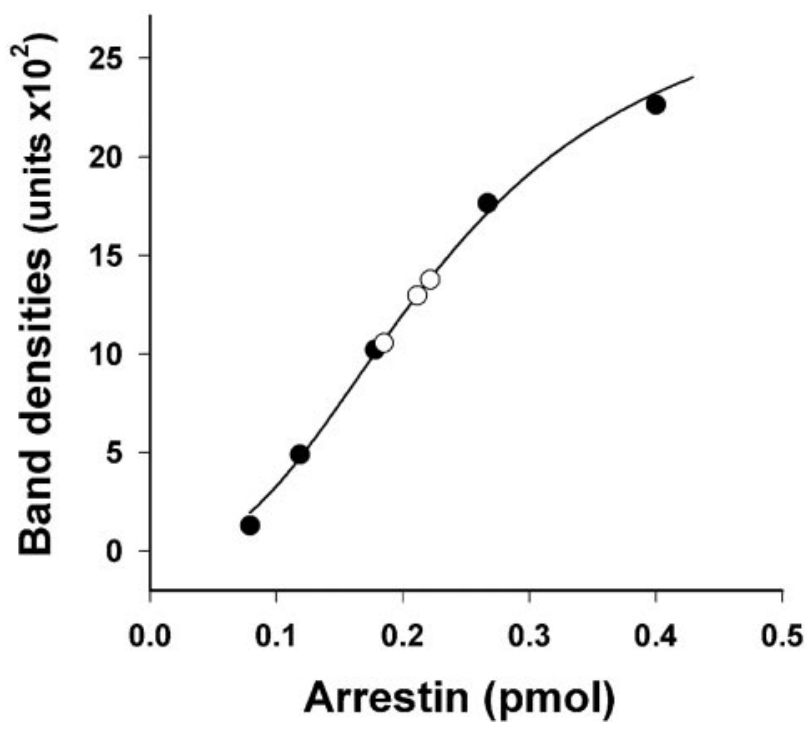

Figure 2. Quantification of arrestin in mouse retinas. $\boldsymbol{A}$, Three wild-type retina extracts containing $0.25 \mathrm{pmol}$ of rhodopsin were analyzed by Western blotting along with five samples containing recombinant mouse arrestin standards premixed with aliquots of retina extract from the arrestin knock-out mouse also containing $0.25 \mathrm{pmol}$ of rhodopsin. $\boldsymbol{B}$, The calibration curve was obtained by plotting the amount of fluorescent signal in each band against the amount of arrestin in the standard (filled circles). The amounts of arrestin in the retina samples (open circles) were determined to be $0.185,0.211$, and $0.222 \mathrm{pmol}$. The data are taken from one of two independent experiments, with the total amount of analyzed retina samples being six.

The amount of translocating arrestin is superstoichiometric to the amount of photoexcited rhodopsin

Based on our quantification of arrestin, the $36 \%$ of the cellular arrestin undergoing the translocation during the $30 \mathrm{~min}$ illumination of the threshold light intensity (Fig. 1) corresponds to $2 \times$ $10^{7}$ molecules. Because $\sim 2 \times 10^{6} \mathrm{R}^{\star}$ was produced by the same light, the amount of translocated arrestin exceeded the amount of photoactivated rhodopsin by $\sim 10$-fold. This stoichiometry reveals that the amount of activated rhodopsin is insufficient to trap an appreciable fraction of arrestin, and therefore, trapping alone cannot explain the translocation phenomenon. Rather, translocation is set by a signaling event triggered as the light intensity reaches a critical level. An appreciable trapping by $\mathrm{R}^{*}$ can occur only at the light intensities significantly above the threshold and could account only for the additional increase in the rod outer segment levels of arrestin observed at light above the threshold level.

We next determined the ratio between the amounts of translocating arrestin and photoexcited rhodopsin immediately after the onset of illumination just above the threshold intensity level $\left(\sim 1400 \mathrm{R}^{\star} / \mathrm{rod} / \mathrm{s}\right)$ (Fig. $\left.3 A\right)$. Arrestin translocation under these conditions was well fit by an exponent with a halftime of $\sim 5 \mathrm{~min}$. The initial rate of arrestin translocation was $\sim 4.5 \%$ of its total cellular content per minute, or $\sim 2.5 \times 10^{6}$ arrestin molecules per minute. Given that the light of this intensity produces $\sim 8.4 \times 10^{4}$ $\mathrm{R}^{\star} / \mathrm{min}$, we calculated the ratio between rhodopsin bleaching and
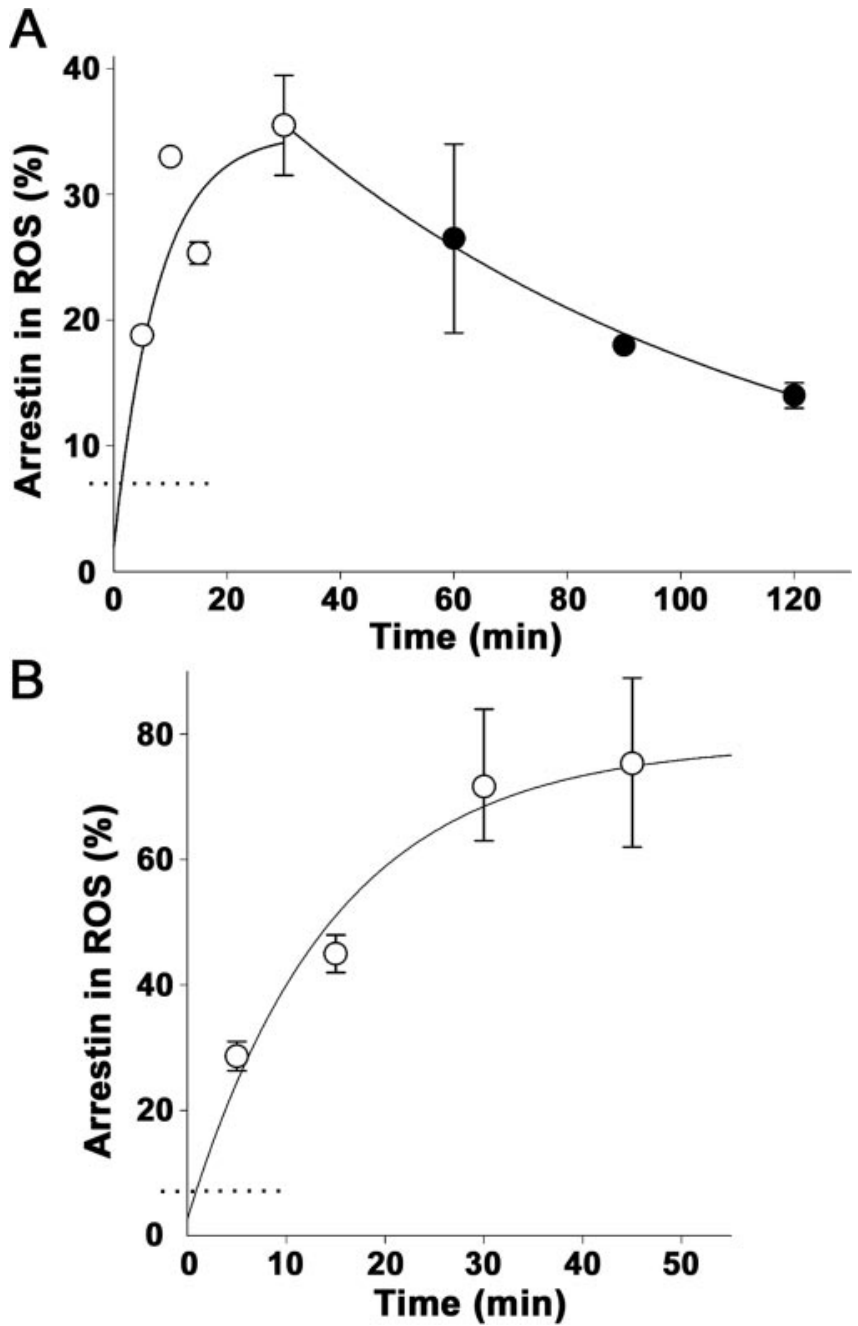

Figure 3. Kinetics of light-dependent arrestin translocation. $A$, Arrestin entry into rod outer segments (ROS) (open circles) was determined in anesthetized mice exposed to continuous light producing $1400 \mathrm{R} / \mathrm{rod} / \mathrm{s}$ using serial sectioning/Western blotting. The exponential curve fitted to the data and originated from the midpoint below the method detectability level (dotted line) indicated that the rate of arrestin translocation was $0.14 \pm 0.05 \mathrm{~min}^{-1}$. Arrestin return to the inner segments in the dark (filled circles) was measured after mice were first illuminated with continuous light of the same intensity for $30 \mathrm{~min}$ and then kept in the dark for various periods of time. The rate of arrestin return calculated from the exponential fit of the data were $0.011 \pm 0.001 \mathrm{~min}^{-1}$. B. Arrestin entry into rod outer segments at bright illumination initially producing $\sim 5 \times 10^{5} \mathrm{R}^{*} / \mathrm{rod} / \mathrm{s}$. The exponential rate of arrestin translocation was $0.072 \pm 0.017 \mathrm{~min}^{-1}$. The number of individual experiments for each time point varied between 2 and 4 ; error bars represent SEM.

arrestin translocation to be $\sim 1: 30$. This number provides a more accurate representation of the arrestin/ $\mathrm{R}^{\star}$ ratio than the 10 -fold value calculated above from Figure 1 because it is taken from the initial linear range of arrestin movement.

\section{Arrestin translocation in the light is more rapid than its return in the dark}

In the next experiment, we examined whether the extent of rhodopsin bleaching affects the rate of arrestin translocation. The translocation time course was measured at a high light intensity $\left(50,000 \mathrm{R}^{\star} / \mathrm{rod} / \mathrm{s}\right)$ that bleached $>90 \%$ rhodopsin during the experiment (Fig. $3 B$ ). The initial translocation rate was only $\sim 20 \%$ higher than in Figure $3 A$, but the total amount of translocated arrestin was approximately twofold larger, shifting the halftime to $\sim 9 \mathrm{~min}$. The increased magnitude of arrestin translocation in 
this experiment is consistent with significant trapping of arrestin by $\mathrm{R}^{\star}$, whereas the similarity between the initial rates provides additional evidence against the arrestin- $\mathrm{R}^{\star}$ interaction being the primary driving force for the translocation.

The extinction of illumination caused arrestin return to the inner segments (Fig. 3A). The return kinetics was determined after the extinction of illumination of the threshold intensity. This allowed us to minimize the effects of arrestin trapping by rhodopsin on the rate of arrestin return. The exponential fit of the data revealed a halftime of $\sim 65 \mathrm{~min}$, which is significantly slower than the rate of arrestin movement in the light-induced direction.

The kinetic parameters identified in our study are generally consistent with those derived from densitometric analysis of immunostained cross sections of the mouse retina (Elias et al., 2004; Nair et al., 2005). However, these other studies reported a twofold to threefold more rapid arrestin translocation in each direction. Elias et al. (2004) also observed a more rapid transducin translocation than we measured previously using serial sectioning with Western blotting (Sokolov et al., 2002) and suggested that the difference originates from a longer time period required to process retinas for serial sectioning than for immunostaining. We disagree because any protein translocation occurring during sample processing would be additive to that completed before the extinction of light, making the formally measured time course faster instead of slower. Rather, we think that this discrepancy reflects the intrinsically nonquantitative nature of the densitometric analysis of immunostaining data. Two untested assumptions of such analyses are that the staining efficiency of a given protein is uniform throughout the cell and that the staining intensity is a linear function of protein concentration. Neither is practical to control using independent protein standards, and these nonlinearities of immunostaining often lead to artifacts of epitope masking and unmasking (cf. Roof and Heth, 1988; Kramer et al., 1997; Ezaki, 2000). This is in contrast to Western blotting, which can be calibrated using purified protein standards.

\section{Arrestin translocation is triggered downstream from phototransduction}

In the final set of experiments, we addressed whether the light dependence of arrestin translocation is conveyed through the conventional phototransduction cascade or through an alternative not-yet-identified mode of light signaling, as suggested for example by Mendez et al. (2003). We did this by studying arrestin translocation in two genetically modified mouse models, the R9AP knock-out and the transducin $\alpha$ subunit knock-out, in which phototransduction was either enhanced or abolished, respectively. The total arrestin content in both mice was normal (data not shown) (for transducin $\alpha$ subunit knock-out, see Calvert et al., 2000).

We first analyzed the R9AP knock-out mouse (Fig. 4), which is characterized by prolonged rod photoresponses caused by a defect in timely deactivation of transducin (Keresztes et al., 2004). The slow recovery of the responses makes these rods significantly more sensitive to steady light than wild-type rods. The dark content of arrestin in the rod outer segments of these mice is low as in wild-type mice. However, exposing R9AP knock-outs to continuous light producing $380 \mathrm{R}^{\star} / \mathrm{rod} / \mathrm{s}$, well below the arrestin translocation threshold in wild-type mice, caused the translocation of $\sim 36 \%$ arrestin. This level of translocation is the same as that caused by the light of threshold intensity $\left(1160 \mathrm{R}^{\star} / \mathrm{rod} / \mathrm{s}\right)$ in wild-type animals.

These data bring us to two conclusions. First, the signal trig-

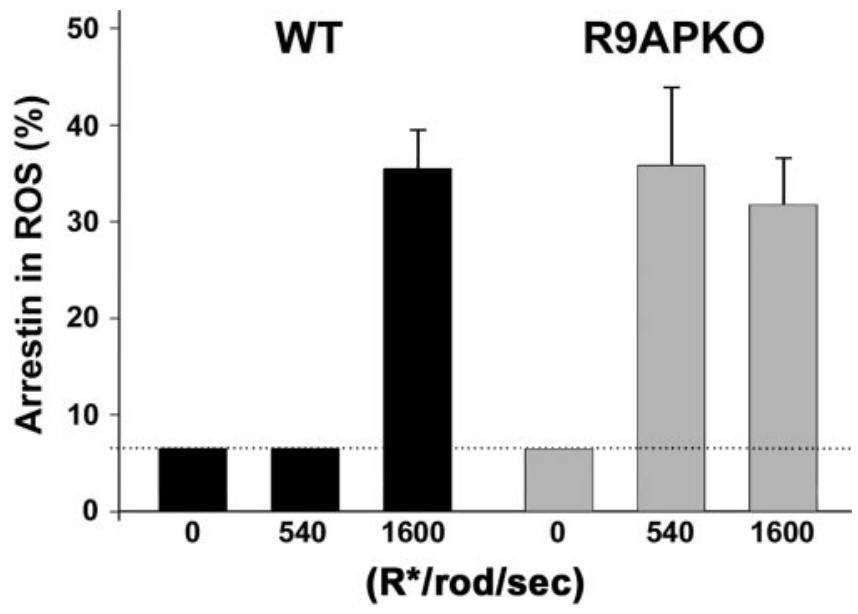

Figure 4. Increased light sensitivity of arrestin translocation in R9AP knock-out mice. Arrestin translocation was analyzed by serial sectioning/Western blotting in wild-type or R9AP knock-out (R9APKO) mice in the dark or after 30 min of continuous illumination at intensities producing 380 or $1160 \mathrm{R}^{*} / \mathrm{rod} / \mathrm{s}$. Error bars represent SEM of the data from three individual experiments. The dotted line indicates the detectability limit of the serial sectioning/Western blotting technique. The bars for the data falling below the 7\% detectability limit are set at $7 \%$; the actual amount of arrestin in rod outer segments (ROS) may be less.

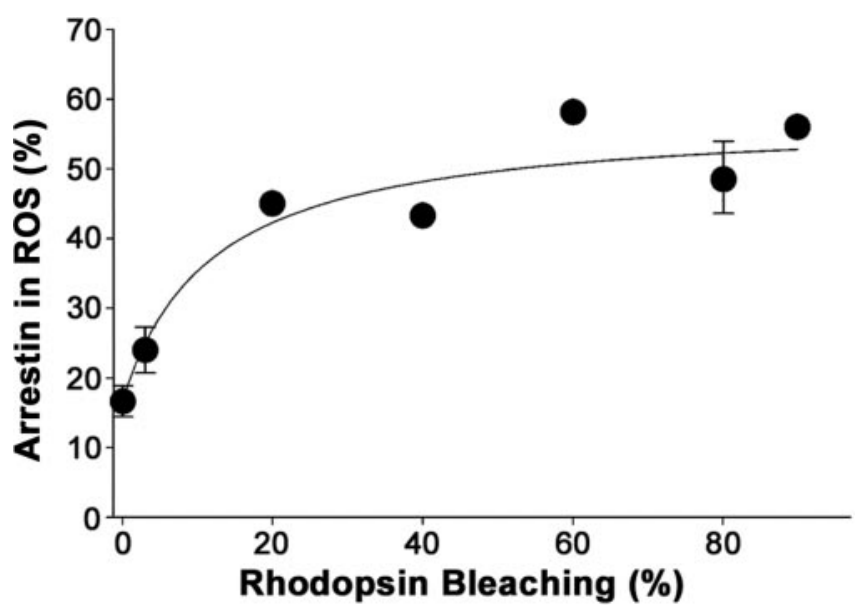

Figure 5. Lack of threshold in arrestin translocation in the transducin $\alpha$ subunit knock-out mouse. Arrestin translocation in transducin $\alpha$ subunit knock-out mice was analyzed by serial sectioning/Western blotting either in the dark or after a 30 min exposure to light of various intensities. The number of individual measurements varied from seven in the dark to between one and three at various light intensities; error bars indicate SEM. ROS, Rod outer segments.

gering arrestin translocation requires conventional phototransduction. Any putative light-sensitive pathway not acting downstream from transducin would not be expected to enhance light sensitivity of arrestin translocation when transducin activity is affected. Second, the light intensity threshold for arrestin translocation is determined by the "amount" of signaling through the phototransduction cascade, not by the amount of rhodopsin bleaching itself.

We next examined arrestin translocation in the transducin $\alpha$ subunit knock-out mouse in which rhodopsin is unable to initiate a photoresponse (Calvert et al., 2000). Two previous studies documented the presence of light-dependent arrestin translocation in this model (Mendez et al., 2003; Zhang et al., 2003b). Data shown in Figure 5 confirm that translocation of arrestin takes place in this mouse but also reveal several major differences from wild-type mice. Unlike in wild-type mice, a significant fraction of 
arrestin $(\sim 17 \%)$ in transducin $\alpha$ knock-outs was found in rod outer segments in the dark. Light of increased intensities caused a gradual increase in this content but only up to $\sim 52 \%$ of the total arrestin amount (compared with $\sim 76 \%$ in Fig. 1 ). Significantly, no threshold was observed in the light-dependency curve.

Given that the total amount of arrestin in this mouse is normal, it appears that, without normal phototransduction, arrestin distribution in the rod is not properly "tuned." Approximately one-half of arrestin remains at its dark location in the inner segments regardless of the conditions of illumination, whereas the other half is evenly distributed throughout the rod cytosol. The latter arrestin pool can be trapped by $\mathrm{R}^{\star}$ at high bleaching levels, resulting in its redistribution to the outer segments observed in Figure 5. The reduced level of arrestin translocation in bright light and lack of threshold in the light-dependency curve further confirm that there is a signaling threshold-dependent event required for arrestin translocation in normal rods.

\section{Discussion}

Light-driven protein translocation has recently become one of the most intensively studied topics in photoreceptor biology. The introduction of quantitative techniques and the availability of genetically manipulated animal models open the door for gaining insights into the nature of this phenomenon. In this study, we used both approaches to describe the most basic characteristics of arrestin translocation in mouse rods: its light dependency, kinetics, and relation to rhodopsin bleaching. This led us to the discovery that the number of translocating arrestin molecules vastly exceeds the number of photoactivated rhodopsins, and that translocation occurs only when a threshold of cascade signaling has occurred. The putative nature of the underlying mechanisms and the physiological consequences of arrestin translocation will be discussed in this section.

\section{Is arrestin translocation driven by diffusion or molecular motors?}

Most of the recent discussion on the mechanisms of protein translocation in rods and cones was centered on whether it is driven by diffusion (cf. Peet et al., 2004; Nair et al., 2005) or by the action of molecular motors carrying arrestin and transducin along axonemal microtubules (cf. McGinnis et al., 2002; Peterson et al., 2005). As we calculated previously for transducin translocation (Strissel et al., 2004), both the observed rate and the volume of protein translocation are much more compatible with diffusion. Data obtained in this study reinforce this argument. With the onset of illumination, up to $5 \%$ of the total arrestin translocates from the inner to the outer segments each minute (Fig. 3). This is equivalent to the rate of $\sim 3,000,000$ molecules per minute, which is nearly 1000 -fold faster than the reported rate of rhodopsin delivery into rod outer segments $[\sim 4300$ molecules per minute (Liu et al., 1999)], viewed as a benchmark for a rapid motor-driven protein transport process. The striking speed of arrestin translocation argues against an active transport mechanism. To the contrary, the calculated rate for the diffusion of an average soluble protein between the rod compartments is severalfold faster than the measured rate of arrestin translocation (for a detailed calculation, see Strissel et al., 2004). Another theoretical argument in favor of diffusion is that its rate is not dependent on the absolute protein concentration in the cell, whereas the capacity of molecular motors could be easily saturated by such vast amounts of proteins as arrestin.

Direct evidence in support of diffusion being rapid enough to underlie protein translocation in rods was obtained in two recent studies (Calvert et al., 2005; Nair et al., 2005). Both demonstrated that soluble green fluorescent protein expressed in rods diffuses between the inner and the outer segments on a timescale of a few minutes, similar to arrestin. Nair et al. (2005) also observed arrestin translocation on a similar timescale within retinas severely depleted of ATP and thus unlikely capable of conducting energydependent protein transport. This observation, however, does not exclude the role of ATP in arrestin translocation under normal conditions, neither does it imply that the basic parameters of arrestin translocation without ATP are normal.

\section{The diffusion model requires arrestin interaction with light- controlled binding sites}

Although the speed of arrestin translocation is consistent with diffusion, diffusion alone cannot account for the observed directionality of this process and the steep arrestin gradients between the inner and outer segments. Therefore, the diffusion model must include additional components that provide this directionality, such as arrestin binding sites controlled by light.

It has been long recognized that arrestin could be "trapped" in the outer segment by the sites provided by photoexcited phosphorylated rhodopsin (Mangini et al., 1994). Conversely, a recent report by Peet et al. (2004) indicates that, in dark-adapted rods, arrestin is confined to unknown binding sites in the inner segment. The latter is evident from the observation that the intracellular distribution of arrestin in the dark does not match that of a soluble green fluorescent protein. One candidate for binding arrestin in the inner segments is the microtubular cytoskeleton, which has been shown to comigrate with arrestin during subcellular fractionation, particularly in the dark (Nair et al., 2004, 2005). In addition, arrestin was reported to colocalize with the microtubular axoneme in Xenopus rods (McGinnis et al., 2002; Peterson et al., 2003), the observation originally proposed as evidence for the involvement of the cytoskeleton in arrestin translocation.

Regardless of the nature of the inner segment sites, they are predicted to have a relatively low affinity for arrestin. This is because a small but critical fraction of arrestin must be present in the free form and available to the outer segments to terminate the photoresponse of dark-adapted rods. The existence of this arrestin fraction is supported by the lack of photoresponse termination in rods of the arrestin knock-out mouse (Xu et al., 1997).

\section{Is the competition between microtubules and rhodopsin sufficient to explain arrestin translocation?}

A version of the diffusion-mediated model of arrestin translocation has been presented in a recent study by Nair et al. (2005). They proposed that the entire phenomenon can be explained by a competition for arrestin binding between the constitutive lowaffinity microtubular sites in the inner segments and the lightinducible high-affinity rhodopsin sites in the outer segments. In the dark, the affinity of microtubules is sufficient for retaining most arrestin in the inner segment compartment, whereas the formation of bleached phosphorylated rhodopsin during illumination rapidly shifts the arrestin distribution equilibrium toward the outer segments. Evidence in support of this hypothesis includes the following: the abolishment of arrestin translocation by hydroxylamine causing rapid $\mathrm{R}^{\star}$ decay, the slowed rate of translocation without rhodopsin phosphorylation, the correlation of arrestin return to the inner segment with rhodopsin dephosphorylation and $\mathrm{R}^{\star}$ decay, and impaired translocation of arrestin mutants with increased affinity to microtubules. The authors con- 
cluded that rhodopsin activation is necessary and sufficient for arrestin translocation.

Although this hypothesis is simple and appealing mechanistically, functionally it makes arrestin translocation an epiphenomenon, because an appreciable translocation occurs only at high bleaching levels when rods are not thought to contribute much to visual perception (Rodieck, 1998) (note that no less than 50\% rhodopsin was bleached in the experiments reported by Nair et al., 2005). Besides, this hypothesis predicts that free arrestin concentration in the outer segments decreases in light, which could not be adaptive for the cell (see below). However, the major challenge for this hypothesis is to meet its quantitative prediction that the amount of bleached rhodopsin is equal to the amount of translocating arrestin. This prediction is not met in our direct measurements. The ratio between the amounts of bleached rhodopsin and translocated arrestin is $\sim 1: 30$ in wild-type rods and even higher in rods of R9AP knock-out mice. Because one $\mathrm{R}^{*}$ molecule cannot bind these quantities of arrestin, our results essentially reject the simple competition hypothesis.

\section{Potential role of arrestin translocation in light adaptation}

Our data indicate that light-mediated arrestin release from storage in the inner segments is not passive but is triggered when the intensity of background illumination approaches the upper limits of the normal range of rod responsiveness (Nakatani et al., 1991; Makous, 2001; Krispel et al., 2003). Because the amount of arrestin translocating at the threshold light intensity significantly exceeds the amount of bleached rhodopsin, the translocation is predicted to lead to an increase in free arrestin concentration in the outer segment cytoplasm. Such an increase could serve two specific functions. First, it could speed the inactivation of individual $\mathrm{R}^{\star}$ molecules, thus improving the temporal resolution of light responses. Second, it could provide the capacity to quench larger amounts of $\mathrm{R}^{*}$ than would be typically encountered by a dark-adapted rod. Although both predictions remain to be evaluated in direct physiological experiments, they are expected to act in addition to an $\sim 10$-fold adaptation effect caused by transducin translocation (Sokolov et al., 2002) because transducin translocation affects the rising phase of the photoresponse, whereas arrestin acts at the level of response recovery.

At high light intensity levels when a significant fraction of rhodopsin becomes bleached, additional arrestin could be translocated to the outer segments as a consequence of its being trapped by $\mathrm{R}^{\star}$ (Fig. 1). As mentioned above, it is difficult to consider this additional translocation an adaptation mechanism because it leads to a decrease, not an increase, in the free arrestin concentration. Rather, it could be viewed as arrestin "utilization" after the actual adaptation phenomenon of light-triggered arrestin release from the inner segments.

In summary, our quantitative analysis makes a major advance in understanding light-dependent arrestin translocation by revealing the key features of this process, its superstoichiometric ratio to $\mathrm{R}^{*}$, and the light-triggered release of arrestin from inner segment storage. This work sets the stage for any future mechanistic studies addressing the molecular nature of this storage, its regulation by light, and the physiological consequences of arrestin release.

\section{References}

Arshavsky VY (2003) Protein translocation in photoreceptor light adaptation: a common theme in vertebrate and invertebrate vision. Sci STKE 2003:PE43.

Arshavsky VY, Lamb TD, Pugh Jr EN (2002) G proteins and phototransduction. Annu Rev Physiol 64:153-187.
Bownds D, Gordon-Walker A, Gaide-Huguenin A-C, Robinson W (1971) Characterization and analysis of frog photoreceptor membranes. J Gen Physiol 58:225-237.

Bradford MM (1976) A rapid and sensitive method for the quantitation of microgram quantities of protein utilizing the principle of protein-dye binding. Anal Biochem 72:248-254.

Brann MR, Cohen LV (1987) Diurnal expression of transducin mRNA and translocation of transducin in rods of rat retina. Science 235:585-587.

Broekhuyse RM, Tolhuizen EF, Janssen AP, Winkens HJ (1985) Light induced shift and binding of S-antigen in retinal rods. Curr Eye Res 4:613-618.

Burns ME, Baylor DA (2001) Activation, deactivation, and adaptation in vertebrate photoreceptor cells. Annu Rev Neurosci 24:779-805.

Calvert PD, Krasnoperova NV, Lyubarsky AL, Isayama T, Nicoló, M, Kosaras B, Wong G, Gannon KS, Margolskee RF, Sidman RL, Pugh Jr EN, Makino CL, Lem J (2000) Phototransduction in transgenic mice after targeted deletion of the rod transducin a-subunit. Proc Natl Acad Sci USA 97:13913-13918.

Calvert PD, Peet JA, Pugh Jr EN (2005) Through the connecting cilium: protein equilibration between the inner and outer segments of rods observed in real time with photoactivatable-GFP [abstract]. Invest Ophthalmol Vis Sci 46:4782.

Coleman JE, Semple-Rowland SL (2005) GC1 deletion prevents lightdependent arrestin translocation in mouse cone photoreceptor cells. Invest Ophthalmol Vis Sci 46:12-16.

Elias RV, Sezate SS, Cao W, McGinnis JF (2004) Temporal kinetics of the light/dark translocation and compartmentation of arrestin and alphatransducin in mouse photoreceptor cells. Mol Vis 10:672-681.

Ezaki T (2000) Antigen retrieval on formaldehyde-fixed paraffin sections: its potential drawbacks and optimization for double immunostaining. Micron 31:639-649.

Fain GL, Matthews HR, Cornwall MC, Koutalos Y (2001) Adaptation in vertebrate photoreceptors. Physiol Rev 81:117-151.

Hardie RC (2003) Phototransduction: shedding light on translocation. Curr Biol 13:R775-R777.

Kassai H, Aiba A, Nakao K, Nakamura K, Katsuki M, Xiong WH, Yau KW, Imai H, Shichida Y, Satomi Y, Takao T, Okano T, Fukada Y (2005) Farnesylation of retinal transducin underlies its translocation during light adaptation. Neuron 47:529-539.

Keresztes G, Martemyanov KA, Krispel CM, Mutai H, Yoo PJ, Maison SF, Burns ME, Arshavsky VY, Heller S (2004) Absence of the RGS9·Gb5 GTPase-activating complex in photoreceptors of the R9AP knockout mouse. J Biol Chem 279:1581-1584.

Kramer I, Hall H, Bleistein U, Schachner M (1997) Developmentally regulated masking of an intracellular epitope of the $180 \mathrm{kDa}$ isoform of the neural cell adhesion molecule NCAM. J Neurosci Res 49:161-175.

Krispel CM, Chen CK, Simon MI, Burns ME (2003) Novel form of adaptation in mouse retinal rods speeds recovery of phototransduction. J Gen Physiol 122:703-712.

Liu X, Udovichenko IP, Brown SD, Steel KP, Williams DS (1999) Myosin VIIa participates in opsin transport through the photoreceptor cilium. J Neurosci 19:6267-6274.

Lyubarsky AL, Daniele LL, Pugh Jr EN (2004) From candelas to photoisomerizations in the mouse eye by rhodopsin bleaching in situ and the light-rearing dependence of the major components of the mouse ERG. Vis Res 44:3235-3251.

Makous W (2001) Scotopic vision. In: Visual neurosciences (Chalupa LM, Werner JH, eds), pp 215-233. Cambridge, MA: MIT.

Mangini NJ, Pepperberg DR (1988) Immunolocalization of 48K in rod photoreceptors. Invest Ophthalmol Vis Sci 29:1221-1234.

Mangini NJ, Garner GL, Okajima T-IL, Donoso LA, Pepperberg DR (1994) Effect of hydroxylamine on the subcellular distribution of arrestin (Santigen) in rod photoreceptors. Vis Neurosci 11:561-568.

McGinnis JF, Matsumoto B, Whelan JP, Cao W (2002) Cytoskeleton participation in subcellular trafficking of signal transduction proteins in rod photoreceptor cells. J Neurosci Res 67:290-297.

Mendez A, Lem J, Simon M, Chen J (2003) Light-dependent translocation of arrestin in the absence of rhodopsin phosphorylation and transducin signaling. J Neurosci 23:3124-3129.

Nair KS, Hanson SM, Kennedy MJ, Hurley JB, Gurevich VV, Slepak VZ (2004) Direct binding of visual arrestin to microtubules determines the 
differential subcellular localization of its splice variants in rod photoreceptors. J Biol Chem 279:41240-41248.

Nair KS, Hanson SM, Mendez A, Gurevich EV, Kennedy MJ, Shestopalov VI, Vishnivetskiy SA, Chen J, Hurley JB, Gurevich VV, Slepak VZ (2005) Light-dependent redistribution of arrestin in vertebrate rods is an energyindependent process governed by protein-protein interactions. Neuron 46:555-567.

Nakatani K, Tamura T, Yau K-W (1991) Light adaptation in retinal rods of the rabbit and two other nonprimate mammals. J Gen Physiol 97:413-435.

Peet JA, Bragin A, Calvert PD, Nikonov SS, Mani S, Zhao X, Besharse JC, Pierce EA, Knox BE, Pugh Jr EN (2004) Quantification of the cytoplasmic spaces of living cells with EGFP reveals arrestin-EGFP to be in disequilibrium in dark adapted rod photoreceptors. J Cell Sci 117:3049-3059.

Peterson JJ, Tam BM, Moritz OL, Shelamer CL, Dugger DR, McDowell JH, Hargrave PA, Papermaster DS, Smith WC (2003) Arrestin migrates in photoreceptors in response to light: a study of arrestin localization using an arrestin-GFP fusion protein in transgenic frogs. Exp Eye Res 76:553-563.

Peterson JJ, Orisme W, Fellows J, McDowell JH, Shelamer CL, Dugger DR, Smith WC (2005) A role for cytoskeletal elements in the light-driven translocation of proteins in rod photoreceptors. Invest Ophthalmol Vis Sci 46:3988-3998.

Philp NJ, Chang W, Long K (1987) Light-stimulated protein movement in rod photoreceptor cells of the rat retina. FEBS Lett 225:127-132.

Pugh Jr EN, Lamb TD (2000) Phototransduction in vertebrate rods and cones: molecular mechanisms of amplification, recovery and light adaptation. In: Handbook of biological physics. Molecular mechanisms in visual transduction (Stavenga DG, DeGrip WJ, Pugh Jr EN, eds), pp 183255. Amsterdam: Elsevier.

Pulvermüller A, Giessl A, Heck M, Wottrich R, Schmitt A, Ernst OP, Choe HW, Hofmann KP, Wolfrum U (2002) Calcium-dependent assembly of centrin-G-protein complex in photoreceptor cells. Mol Cell Biol 22:2194-2203.
Rodieck RW (1998) The first steps in seeing. Sunderland, MA: Sinauer.

Roof DJ, Heth CA (1988) Expression of transducin in retinal rod photoreceptor outer segments. Science 241:845-847.

Sokolov M, Lyubarsky AL, Strissel KJ, Savchenko AB, Govardovskii VI, Pugh Jr EN, Arshavsky VY (2002) Massive light-driven translocation of transducin between the two major compartments of rod cells: a novel mechanism of light adaptation. Neuron 34:95-106.

Sokolov M, Strissel KJ, Leskov IB, Michaud NA, Govardovskii VI, Arshavsky VY (2004) Phosducin facilitates light-driven transducin translocation in rod photoreceptors: evidence from the phosducin knockout mouse. J Biol Chem 279:19149-19156.

Strissel KJ, Sokolov M, Arshavsky VY (2004) Light-dependent translocation of signaling proteins in vertebrate and invertebrate photoreceptors. In: Recent advances in human biology (Williams, DS, ed), pp 163-193. River Edge, NJ: World Scientific.

Strissel KJ, Lishko PV, Trieu LH, Kennedy MJ, Hurley JB, Arshavsky VY (2005) Recoverin undergoes light-dependent intracellular translocation in rod photoreceptors. J Biol Chem 280:29250-29255.

Whelan JP, McGinnis JF (1988) Light-dependent subcellular movement of photoreceptor proteins. J Neurosci Res 20:263-270.

Xu J, Dodd RL, Makino CL, Simon MI, Baylor DA, Chen J (1997) Prolonged photoresponses in transgenic mouse rods lacking arrestin. Nature 389:505-509.

Zhang H, Cuenca N, Ivanova T, Church-Kopish J, Frederick JM, MacLeish PR, Baehr W (2003a) Identification and light-dependent translocation of a cone-specific antigen, cone arrestin, recognized by monoclonal antibody 7G6. Invest Ophthalmol Vis Sci 44:2858-2867.

Zhang H, Huang W, Zhang H, Zhu X, Craft CM, Baehr W, Chen CK (2003b) Light-dependent redistribution of visual arrestins and transducin subunits in mice with defective phototransduction. Mol Vis 9:231-237.

Zhu X, Li A, Brown B, Weiss ER, Osawa S, Craft CM (2002) Mouse cone arrestin expression pattern: light induced translocation in cone photoreceptors. Mol Vis 8:462-471. 